Abstract P74 Table 1 Prevalence of specific antibody deficiency and associated patient characteristics

\begin{tabular}{|c|c|c|c|c|c|c|c|}
\hline & Prevalence & $\begin{array}{l}\text { Bronchial wall thickening } \\
\text { on CT chest }\end{array}$ & $\begin{array}{l}\text { Bronchiectasis on } \\
\text { СT chest }\end{array}$ & $\begin{array}{l}\geq 7 \text { Exacerbations } \\
\text { past year }\end{array}$ & $\begin{array}{l}\text { Total ITU } \\
\text { admissions }\end{array}$ & $\begin{array}{l}\text { Percentage predicted } \\
\text { FEV1 }(\%)\end{array}$ & $\begin{array}{l}\text { Inhaled steroid (BDP } \\
\text { equivalent) } \mu \mathrm{g}\end{array}$ \\
\hline$n=45$ & $\mathrm{n}(\%)$ & n (\%) & $\mathrm{n}(\%)$ & $\mathrm{n}(\%)$ & $\mathrm{n}(\%)$ & Mean (SD) & Mean (SD) \\
\hline $\begin{array}{l}\text { Total } H \text {. influenzae } \\
\text { deficient }\end{array}$ & $30(70)$ & & & & & & \\
\hline $\begin{array}{l}\text { H. influenzae deficient } \\
\text { alone }\end{array}$ & $13(31)$ & $8(23)$ & $5(5)-14$ & $4(3)-17$ & $3(3)-18$ & $57(16)$ & $2540(2240)$ \\
\hline $\begin{array}{l}\text { Total S. pneumoniae } \\
\text { deficient }\end{array}$ & $23(51)$ & & & & & & \\
\hline $\begin{array}{l}\text { S. pneumoniae deficient } \\
\text { alone }\end{array}$ & $5(12)$ & $3(8.5)$ & $2(2)-6$ & $1(0)-4$ & $0(2)-0$ & 77 (8) & 1933 (1101) \\
\hline Combined Deficiency & $17(40)$ & $11(31)$ & $6(10)-17$ & $8(3)-33$ & $4(2)-24$ & $73(23)$ & $1837(900)$ \\
\hline Competent Specific & $7(13)$ & $2(6)$ & $3(2)-8.5$ & $2(3)-6$ & $0(3)-0$ & $70(32)$ & $1840(607)$ \\
\hline Antibody Levels & & & & & & & \\
\hline
\end{tabular}

significantly reduced lung function in severe asthma patients with specific antibody deficiency. The prevalence and impact of specific antibody deficiency in this patient group is not known. Aim We aimed to determine the prevalence of specific antibody deficiency and its association with markers of disease severity.

Methods We prospectively collected data from all new patients attending the regional severe asthma clinic. We recorded demographic details and markers of disease severity including: BTS treatment step, inhaled corticosteroid (ICS) dose, spirometry, blood and sputum eosinophil count, radiological findings such as bronchiectasis and bronchial wall thickening, exacerbations in the last year and ITU admissions. Specific antibody levels to Haemophilus Influenzae (Streptococcus Pneumoniae $(<0.35 \mu \mathrm{g} /$ $\mathrm{ml}$ to at least 6 of the 12 serotypes classed as deficient) were measured.

Results Data for 53 patients (39F), mean (SD) age 49.6 (15.9) years were available. Mean (SD) FEV1 was 69 (22)\% predicted, ICS dose 1914 (1337) micrograms, and BMI 31.5 (8.6) kg/m². All were at BTS step 3-5. Information on specific antibody levels was available for 43 and 45 patients for $H$ Influenzae and $S$ pneumoniae respectively and for both in 42 patients. Overall out of the 42 patients $35(83 \%)$ were deficient to one or both the organisms. Of these 13 (31\%) were deficient to H Influenzae alone, 5(12\%) to $S$ pneumoniae alone, and 17 (40.5\%) to both. Looking at each organism separately $30(70 \%)$ out of 43 were deficient to $H$ Influenzae and 23 out of 45 (51\%) were deficient to $S$ pneumoniae. Of the 32 patients for whom data were available $20(63 \%)$ had 7 or more exacerbations in the preceding year and two thirds of these had bronchial wall thickening on their CT scans. A third of patients (30\%) reported at least one ITU admission. The presence of specific antibody deficiency did not correlate with any clinical or radiological findings.

Conclusion Specific antibody deficiency to $H$ influenza and $S$ pneumoniae is remarkably common in moderate to severe asthma. Further studies are required to determine the clinical significance of this finding.

\section{P75 CLUSTER CLASSIFICATION AS A PREDICTOR OF ADVERSE RADIOLOGICAL OUTCOMES IN ALLERGIC FUNGAL AIRWAYS DISEASE (AFAD)}

K Woolnough, C Newby, M Richardson, AJ Wardlaw. Department of Infection, Inflammation and Immunity, Institute for Lung Health, University of Leicester, Leicester, UK

10.1136/thoraxjnl-2015-207770.212
Introduction The relationship between immunological biomarkers and evidence of lung damage has not been established in asthmatics that are sensitised to fungi. We sought to determine what features of allergic fungal airways disease were related to adverse radiological outcomes by the use of cluster analysis.

Method Factor analysis was used to determine the significance of different clinical and immunological variables, the number of clusters present and cluster membership $(n=423)$. The presence of radiological indicators of lung damage and inflammation were then assessed between these groups.

Results Three clusters were identified. Cluster 1 (37.1\%) were obese, had late onset and minimal eosinophilic disease, cluster 2 (40.9\%) had late onset eosinophilic disease and cluster 3 (22\%) had early onset, atopic disease. Sensitisation to A. fumigatus was more prevalent in cluster $3(94.2 \%$; sIgE A. fumigatus $10.2 \mathrm{kUA} /$ L (1.31-35.4)), compared to cluster 1 (44.9\%; sIgE A. fumigatus $0.16 \mathrm{kUA} / \mathrm{L}(0.03-0.94))$ and cluster 2 (45.7\%; sIgE A. fumigatus $0.25 \mathrm{kUA} / \mathrm{L}(0.06-1.36))$.

Cluster 3 had a greater degree of airflow obstruction $(\mathrm{p}<$ $0.001)$, bronchiectasis $(69 \%, \mathrm{p}<0.001)$, tree in bud $(32.4 \%, \mathrm{p}$ $<0.001)$, collapse/consolidation (48.6\%, p0.007) and fibrosis $(31.7 \%, \mathrm{p}<0.05)$ than any of the other groups.

Conclusion This cluster analysis demonstrates that sensitisation to A. fumigatus, in addition to the other known clinical phenotypes, identifies asthmatics most at risk of developing fixed airflow obstruction and radiological features of airway inflammation and damage.

\section{P76 PSYCHOGENIC VOICE DISORDER MIMICKING TREATMENT-REFRACTORY RESPIRATORY DISEASE}

1J Selby, ${ }^{2} \mathrm{G}$ Sandhu, ${ }^{1} \mathrm{G}$ Scadding, ${ }^{1} \mathrm{~A}$ Menzies-Gow, ${ }^{1} \mathrm{JH}$ Hull. ${ }^{1}$ Royal Brompton Hospital, London, UK; ${ }^{2}$ Charing Cross Hospital, London, UK

\subsection{6/thoraxjnl-2015-207770.213}

Introduction and objectives Psychogenic voice disorder (PVD) is widely acknowledged by voice and ENT specialists as an important cause of dysphonia and breathlessness. The significance of abnormal vocal function in the aetiology of respiratory symptoms is under-recognised in respiratory medicine. The aim of this study is to highlight the importance of PVD as a key differential diagnosis for patients who present with respiratory symptoms and altered voice quality. 
Methods We retrospectively reviewed referrals with respiratory symptoms and dysphonia to the upper airway service at the Royal Brompton, over a 12 month period to 2015. PVD was identified according to accepted criteria: ${ }^{1}$ no structural or neurological laryngeal disease, discrepancy between laryngeal status and voice quality, temporary loss of volitional control over phonation, (e.g. frequently reported as secondary to dyspnoea), normal voicing on vegetative manoeuvres (e.g. coughing) and positive psychological factors associated with onset of symptoms. Perceptual voice quality was rated using the GRBAS scale.

Results Ten female patients were identified as having PVD (70\% type $2,20 \%$ type $3,10 \%$ type 1 ). All patients had preserved spirometric indices but daily symptoms of dyspnoea and dysphonia. Respiratory diagnoses at referral included chronic cough (20\%), difficult asthma (50\%) and unexplained dyspnoea (30\%), with symptoms of between 2 months and 15 years' duration. The majority of patients (70\%) were receiving treatment with either oral $+/$ - inhaled corticosteroid prior to referral. Perceptual voice quality varied among patients, but in all cases normal voice was restored by the end of the first treatment session, leading to subjective reduction in breathlessness. Relevant psychological factors were identified as an underlying cause of the voice disorder.

Conclusion PVD is an under-recognised cause of treatmentrefractory respiratory symptoms in patients with altered voice quality. Prior to referral, these symptoms are often attributed to the use of inhaled corticosteroid, yet accurate diagnosis and targeted therapy permits rapid restoration of normal voice and symptomatic improvement. This case series underpins the importance of collaborative working between SLT and respiratory medicine to ensure patients receive timely and appropriate specialist treatment.

\section{REFERENCE}

1 Butcher P, Elias A, Cavalli L. Understanding and treating psychogenic voice disorder: A CBT framework, 2007

\section{P77 HYPOXIC CHALLENGE TESTING FOR FITNESS TO FLY IN SEVERE ASTHMA}

CM Orton, PM George, S Ward, A Menzies-Gow, JH Hull. The Royal Brompton, London, UK

10.1136/thoraxjnl-2015-207770.214

Introduction and objectives Commercial airline travel poses a recognised risk to patients with respiratory disease, including in those with asthma. Hypoxic challenge testing (HCT) is typically employed to mitigate this risk by dictating in-flight oxygen requirement. The objective of this work was to evaluate the role of HCT in patients with severe asthma.

Methods A retrospective analysis was performed of all BTS/ SIGN Asthma Step 5 adult individuals under the Royal Brompton Hospital severe asthma service, who completed HCT between 2007 and 2014. In line with British Thoracic Society recommendations, under hypoxic conditions a reduction in $\mathrm{PaO}_{2}$ to $<6.6 \mathrm{kPa}$ was reported as a positive result. $\mathrm{A} \mathrm{PaO}_{2}$ level of 6.6-7.5 kPa was considered borderline and supplemental oxygen was advised if co-existent evidence of hyperventilation. Electrocardiograph monitoring was performed in all patients during the HCT.

Results Of the 37 patients studied, 21 (57\%) had a positive HCT. Individuals with a positive HCT had a lower $\mathrm{PaO}_{2}$ under normoxic conditions (10.1 kPa v $11.4 \mathrm{kPa}, \mathrm{p}<0.01)$, but similar $\mathrm{PaCO}_{2}$ level (4.80 kPa v $\left.4.91 \mathrm{kPa}, \mathrm{p}>0.05\right)$. Baseline oxygen saturation was poorly predictive of the need for supplementary oxygen and two-thirds of patients, for whom supplementary oxygen was recommended, had a baseline $\mathrm{SpO}_{2}$ level of greater than 95\%; approximately half of these individuals desaturated to less that $90 \%$ on HCT (Figure 1). Lung function was more obstructed in the positive HCT group (predicted FEV1 (52\% v 78\%, p < 0.01). Across the entire cohort, HCT was associated with a mean rise in heart rate (HR) of $5 \mathrm{bpm}$ and there was no evidence of dysrhythmia or change in QTc. A combination of any two of: baseline $\mathrm{PaO}_{2} \leq 10.5 \mathrm{kPa}$, FEV1 $\leq 60 \%$ predicted and PEF $\leq 350 \mathrm{~L} / \mathrm{min}$ predicted the need for in-flight oxygen with a sensitivity of $90 \%$ and a specificity of $69 \%$.

\section{$\mathrm{SaO}_{2}$ in patients requiring $\mathrm{O}_{2}$}

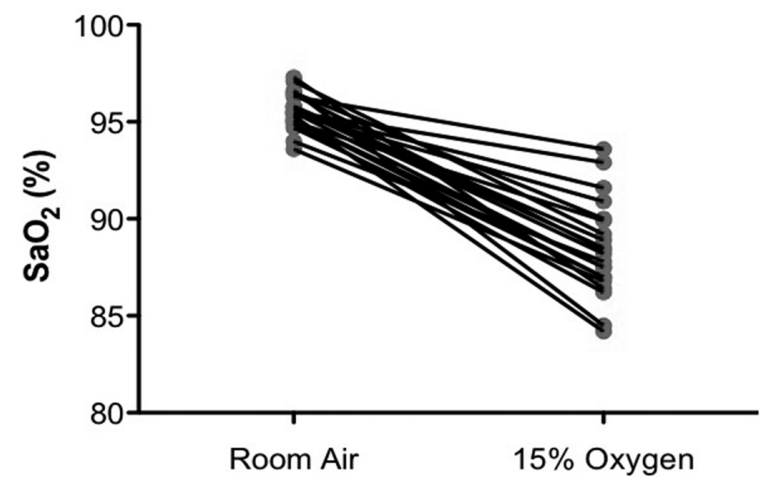

\section{Abstract P77 Figure 1}

Conclusions In patients with severe asthma, baseline oxygen saturation level is poorly predictive of the need for in-flight oxygen. Our findings indicate that a HTC should be considered for all BTS/SIGN Step 5 asthmatics in whom air travel is being considered and should certainly be recommended in those with impaired lung function.

\section{P78 STUDY OF MORTALITY IN SEVERE AND DIFFICULT TO TREAT ASTHMA}

I Sullivan, K O'Shea, A Mansur. Heartlands Hospital, Birmingham, UK

\subsection{6/thoraxjnl-2015-207770.215}

Introduction The National Report on Asthma Death (NRAD 2014) highlighted important shortcomings related to asthma management and an important number of patients still die from asthma. However, the mortality rate and causes of mortality in the severe asthma services has not been previously reported.

Aim To study what patients with severe asthma die from and what is their risk of mortality

Methods All patients attending our severe asthma service who had died between March 2009 and December 2014 were identified. We retrieved data from case notes, GPs, local hospitals and local database using a pre designed proforma which included cause of death, place of death, age at time of death, clinical details on asthma duration, lung function, biomarkers, medication, exacerbations including hospitalisation and co-morbidities. Causes of death was obtained from death certificates and when available coroner's post-mortem reports.

Results Of the 520 patients attended our service between January 2009 and December 2014, there were 24 deaths (4.6\% over 72 months, $0.7 \%$ annually). The mean age of death was $51 \mathrm{yrs}$ (range 21-69), 17/24 (71\%) were females. 50\% had poorly 